\section{Mycobacterium mageritense Lymphadenitis in Child}

\author{
Miguel García-Boyano, Fernando Baquero-Artigao, \\ Carlos Toro, Marina Alguacil-Guillén, \\ Fernando Lázaro-Perona, Cristina Calvo
}

\begin{abstract}
Author affiliation: Hospital Universitario La Paz, Madrid, Spain (M. García-Boyano, F. Baquero-Artigao, C. Toro, M. Alguacil-Guillén, A. Lázaro-Perona, C. Calvo); Translational Research Network of Pediatric Infectious Diseases, Madrid (F. Baquero-Artigao, C. Calvo); CIBERINFEC, Instituto de Salud Carlos III, Madrid (F. Baquero-Artigao, C. Calvo)

DOI: https://doi.org/10.320/eid2803.211486
\end{abstract}

Although human infections caused by Mycobacterium mageritense are rare, there are some case reports involving sinusitis, pneumonia, and hospital-acquired infections in adults. We report a case of lymphadenitis caused by M. mageritense in a child in Spain.

$M$ ycobacterium mageritense was identified as a novel distinct species in 1997. Its name is derived from Magerit, the old Arabic name of Madrid, Spain, the source of most of the human sputum specimens from which it was first isolated (1). Five years later, cases of clinical disease caused by $M$. mageritense were reported in adults (2). We report a case of lymphadenitis caused by $M$. mageritense in a child in Spain.

A previously healthy boy, 2 years and 9 months of age, came to a pediatric clinic because of a 1-week history of swelling of the right submandibular lymph node. Physical examination showed lymph node swelling in the right submandibular region with redviolet discolored skin. He did not had a fever, pain, or any other signs and symptoms. An ultrasound examination showed an enlarged submandibular lymph node $18 \mathrm{~mm}$ in diameter. Laboratory studies showed a leukocyte count of 9,220 cells $/ \mathrm{mm}^{3}$ (reference range $4,800-15,000$ cells $/ \mathrm{mm}^{3}$ ), a differential count of $42 \%$ neutrophils (reference range $55 \%-70 \%$ ), and a C-reactive protein level of $<0.05 \mathrm{mg} / \mathrm{dL}$ (reference range $<1-0.5 \mathrm{mg} / \mathrm{dL}$ ).

Three days later, he underwent fine-needle aspiration of the involved lymph node. Histopathologic analysis showed necrotizing granulomatous lymphadenitis. Acid-fast bacillus staining was negative. Therefore, a nontuberculous mycobacterial lymphadenitis was suspected and treatment with oral clarithromycin (7.5 mg/kg every $12 \mathrm{~h}$ ) and ciprofloxacin (15 mg/kg every $12 \mathrm{~h}$ ) was started.
A rapidly growing mycobacterium was isolated from the lymph node specimen after 6 days of incubation in liquid culture medium (BBL Mycobacteria Growth Indicator Tube; Becton Dickinson, https:/ / www.bd.com). It was identified as $M$. mageritense by using GenoType Mycobacterium CM version 2.0 (Hain Lifescience, https://www.hain-lifescience. de). Matrix-assisted laser desorption/ionization time-of-flight mass spectrometry (Bruker Daltonics, https:/ / www.bruker.com) yielded a score of 2.4 for $M$. mageritense. Whole-genome sequencing was performed to confirm these findings (GenBank accession no. JAJJNE010000000).

Susceptibility testing using a microdilution technique showed a susceptible MIC for linezolid ( $8 \mu \mathrm{g} /$ $\mathrm{mL})$; an intermediate MIC for moxifloxacin $(2 \mu \mathrm{g} /$ $\mathrm{mL})$, imipenem $(8 \mu \mathrm{g} / \mathrm{mL})$, and cefoxitin $(32 \mu \mathrm{g} / \mathrm{mL})$; and a resistant MIC for trimethoprim/sulfamethoxazole $(>8 / 152 \mu \mathrm{g} / \mathrm{mL})$, ciprofloxacin $(>4 \mu \mathrm{g} / \mathrm{mL})$, amikacin $(>64 \mu \mathrm{g} / \mathrm{mL})$, clarithromycin $(>16 \mu \mathrm{g} / \mathrm{mL})$, doxycycline $(>16 \mu \mathrm{g} / \mathrm{mL})$, and tobramycin $(>16 \mu \mathrm{g} /$ $\mathrm{mL})$. Breakpoints were those suggested by the Clinical and Laboratory Standards Institute for rapidly growing mycobacteria (3).

Accordingly, 3 weeks after fine-needle aspiration was performed, clarithromycin was replaced by oral linezolid ( $10 \mathrm{mg} / \mathrm{kg}$ every $8 \mathrm{~h}$ ). However, this change was promptly stopped because of intolerance to linezolid, and clarithromycin was given again. The enlarged lymph node gradually improved, and antimicrobial drug treatment was discontinued 11 weeks after initial prescription. The lymph node was reduced to $<50 \%$ of its initial size. Complete excision of residual lymph node and scar tissue was performed 2 months later, leading to total resolution of the lymphadenitis.

The biochemical and drug susceptibility patterns of $M$. mageritense are relatively similar to the formerly known $M$. fortuitum third biovariant complex $(1,2)$. It is not surprising that they also seem to have the same clinical spectrum (2). Although human infections caused by $M$. mageritense are rare, there are case reports involving sinusitis, pneumonia, and hospitalacquired infections, including catheter-related bloodstream infections, implantable cardioverter defibrillator-related infections, prosthetic valve endocarditis, and intrathecal catheter-related meningitis $(2,4,5)$. Skin and soft tissue infections, including parotitis, furunculosis, and surgical site infections, have also been reported (4).

Mycobacteria are widespread in nature (1) and rapidly growing mycobacteria, such as $M$. mageritense, are ubiquitous in most municipal water supplies (6). 
Although M. mageritense has been isolated from cutaneous lesions of a tsunami survivor (7) and from 2 patients who received footbaths at the same nail salon (6), in most of these case reports, such as for our case, the source of contamination was unknown. Thus, $M$. mageritense is a rapidly growing mycobacteria that can cause granulomatous lymphadenitis in children. Clinicians should be aware of this bacteria during differential diagnoses.

\section{Acknowledgment}

We thank Jaime Esteban for providing assistance with antimicrobial drug susceptibility testing.

\section{About the Author \\ Dr. García-Boyano is a doctoral candidate in pediatric infectious diseases at the Hospital Universitario La Paz, Madrid. His primary research interests are tropical medicine, HIV, and healthcare-associated infections.}

\section{References}

1. Domenech P, Jimenez MS, Menendez MC, Bull TJ, Samper S, Manrique A, et al. Mycobacterium mageritense sp. nov. Int J Syst Bacteriol. 1997;47:535-40. https:/ / doi.org/ 10.1099/00207713-47-2-535

2. Wallace RJ Jr, Brown-Elliott BA, Hall L, Roberts G, Wilson RW, Mann LB, et al. Clinical and laboratory features of Mycobacterium mageritense. J Clin Microbiol. 2002;40:29305. https:// doi.org/10.1128/JCM.40.8.2930-2935.2002

3. Clinical and Laboratory Standards Institute. Performance standards for susceptibility testing of Mycobacteria, Nocardia spp, and other aerobic Actonomycetes. CLSI supplement M62. Wayne (PA): The Institute; 2018.

4. Okabe T, Sasahara T, Suzuki J, Onishi T, Komura M, Hagiwara S, et al. Mycobacterium mageritense parotitis in an immunocompetent adult. Indian J Microbiol. 2018;58:28-32. https://doi.org/10.1007/s12088-017-0692-y

5. Kim J, Seong MW, Kim EC, Han SK, Yim JJ. Frequency and clinical implications of the isolation of rare nontuberculous mycobacteria. BMC Infect Dis. 2015;15:9. https:/ / doi.org/ 10.1186/s12879-014-0741-7

6. Gira AK, Reisenauer AH, Hammock L, Nadiminti U, Macy JT, Reeves A, et al. Furunculosis due to Mycobacterium mageritense associated with footbaths at a nail salon. J Clin Microbiol. 2004;42:1813-7. https:/ / doi.org/10.1128/ JCM.42.4.1813-1817.2004

7. Appelgren P, Farnebo F, Dotevall L, Studahl M, Jönsson B, Petrini B. Late-onset posttraumatic skin and soft-tissue infections caused by rapid-growing mycobacteria in tsunami survivors. Clin Infect Dis. 2008;47:e11-6. https://doi.org/10.1086/589300

Address for correspondence: Miguel García-Boyano, C/ Ginzo de Limia $55,9^{\circ} \mathrm{C}$, Madrid 28034, Spain; email: miguelgarciaboyano@gmail.com

\section{SARS-CoV-2 Breakthrough Infections after Introduction of 4 COVID-19 Vaccines, South Korea, 2021}

\author{
Seonju Yi, ${ }^{1}$ Young June Choe, ${ }^{1}$ Jia Kim, \\ Yoo-Yeon Kim, Ryu Kyung Kim, Eun Jung Jang, \\ Do Sang Lim, Hye Ryeon Byeon, Sangwon Lee, \\ Eonjoo Park, Seung-Jin Kim, Young-Joon Park
}

Author affiliations: Korea Disease Control and Prevention Agency, Cheongju, South Korea (S. Yi, J. Kim, Y.-Y. Kim, R.K. Kim, E.J. Jang, D.S .Lim, H.R. Byeon, S. Lee, E. Park, S.-J. Kim, Y.-J. Park); Korea University Anam Hospital, Seoul, South Korea (Y.J. Choe)

DOI: https://doi.org/10.3201/eid2803.212210

We conducted a nationwide retrospective cohort study to estimate severe acute respiratory syndrome coronavirus 2 (SARS-CoV-2) breakthrough infection among recipients of 4 different vaccines in South Korea. Age-adjusted breakthrough infection rate per month was highest for Janssen (42.6/100,000 population), followed by AstraZeneca (21.7/100,000 population), Pfizer-BioNTech (8.5/100,000 population), and Moderna (1.8/100,000 population).

Since their rollout, vaccines have been highly effective globally in controlling coronavirus disease (COVID-19), caused by severe acute respiratory syndrome coronavirus 2 (SARS-CoV-2) (1). Breakthrough infections have been reported in some vaccine recipients, suggesting the need for public health assessment and monitoring (2). To date, the vaccine-specific data on breakthrough infections are limited. In early 2021, the national immunization program of South Korea introduced 4 COVID-19 vaccines: ChAdOx1 nCov-19 (AstraZeneca, https://www.astrazeneca. com), BNT162b2 (Pfizer-BioNTech, https://www. pfizer.com), Ad26.COV2.S (Johnson \& Johnson/Janssen [hereafter Janssen], https://www.janssen.com), and mRNA-1273 (Moderna, https:/ / www.moderna. com). As of October 10, 2021, a total of $70 \%$ of the country's population have received $\geq 1$ dose of vaccine (3). Introduction of the vaccines provided an opportunity to study breakthrough infections by different vaccine types. We describe a snapshot of SARSCoV-2 breakthrough infections in South Korea and aim to identify risk by age group that might influence the observed pattern.

We conducted a nationwide retrospective cohort study to estimate SARS-CoV-2 breakthrough

${ }^{1}$ These authors contributed equally to this article. 